\title{
High resolution computed tomography in the evaluation of interstitial lung diseases: Imaging perspective revisited with review of literature
}

\author{
Gupta $S^{1}$, Mohd Ilyas ${ }^{2}$, Gupta $V^{3}$, Dev G ${ }^{4}$ \\ ${ }^{1}$ Dr Shivani Gupta, Senior Resident, ${ }^{2}$ Dr Mohd Ilyas, Postgraduate, ${ }^{3}$ Dr Vikrant Gupta, Lecturer; ${ }^{4}$ Dr Ghanshyam Dev, \\ Professor and Head: all authors are affiliated with Department of Radiodiagnosis and Imaging, Govt. Medical College \\ Jammu, Jammu and Kashmir, India
}

Address for Correspondence: Dr Mohd Ilyas, Department of Radiodiagnosis and Imaging, Govt. Medical College Jammu, Jammu and Kashmir, India, email: ilyasmir40@gmail.com

\begin{abstract}
Aims and Objectives: The study was designed with a broad objective of studying the imaging findings of the common interstitial lung diseases and to assess the severity of the disease and determine the reversibility capability of the pulmonary parenchymal damage in ILD's. Patients and Methods: This was a prospective, observational study in which we evaluated 50 patients suspected of having interstitial lung diseases based on radiographic or clinical findings, by high resolution computed tomography (HRCT) over a period of one year. Results: Out of the 50 patients, majority of them had idiopathic pulmonary fibrosis $(n=16)$ followed by interstitial pulmonary involvement in patients of rheumatoid arthritis $(n=13)$, scleroderma $(n=10)$ and systemic lupus erythematosis $(n=9)$. The less common ILD's included were Occupational lung disease $(n=1)$ and Mycosis fungoides $(n=1)$. Conclusion: HRCT of the lung in cases of the suspected interstitial lung diseases forms an invaluable tool for accurate and early identification and in conjunction with the clinical findings can obviate the need of lung biopsy in diagnosis of ILD's.
\end{abstract}

\section{Introduction}

Interstitial lung diseases (ILD) also called as diffuse infiltrative lung diseases are heterogeneous group of disorders that predominantly affect the lung parenchyma and are characterized by alveolar, septal thickening, fibroblast proliferation and pulmonary fibrosis. Although over 100 distinct entities of ILD are recognized, idiopathic pulmonary fibrosis, sarcoidosis and connective tissue disease related ILD account for most of ILD. The prevalence of the IPF is around 54 per 100000 adults using broad case definition and 17 per 100000 using narrow case definition. Although ILDs are more commonly seen in adults, some forms such as hypersensitivity pneumonitis and idiopathic interstitial pneumonias are encountered in children [1]. Patients with ILD most commonly present with shortness of breath with exertion, fatigue, weakness, loss of appetite, loss of weight, dry cough and discomfort in chest. These people have a diffuse infiltrative pattern on chest radiograph [2]. This study is aimed at the imaging

Manuscript received: $02^{\text {nd }}$ April 2017

Reviewed: $10^{\text {th }}$ April 2017

Author Corrected: $19^{\text {th }}$ April 2017

Accepted for Publication: 30 ${ }^{\text {th }}$ April 2017 features of various common interstitial lung diseases so that the diagnosis is made early and irreversible complications avoided.

Interstitial lung diseases are classified into those with known causes and with unknown causes. Those with known causes include Connective tissue disease associated ILD, Pneumoconiosis, Drug-induced, Smoking-related ILD, Radiation-induced and Toxic inhalation-induced ILD. Those with unknown causes include Idiopathic pulmonary fibrosis, Sarcoidosis, Pulmonary lymphangioleiomyomatosis and Pulmonary alveolar proteinosis [1]. Interstitial lung diseases are manifested radiographically as either reticular pattern, nodular pattern or reticulonodular pattern. Interlobular septae contains lymphatics and veins along with connective tissue. Diseases that involve these structures will result in thickened interlobular septae and irregular appearances of the pleural surfaces like in IPF, lymphangitis carcinomatosis and pulmonary edema [3]. 
For many years, the plain chest radiograph was the only imaging used in the diagnosis of interstitial lung diseases. With the advent of CT, came the first opportunity to assess gross lung structure. Conventional 8-10 mm collimation scans allowed better assessment of lung parenchyma. However CT only played minor role in diagnosis of interstitial lung diseases until the introduction of High Resolution Computed Tomography (HRCT). By eliminating superimposition of structures, CT allows for a better assessment of the type, distribution and severity of parenchymal abnormalities [4]. Since its introduction in the 1980s, HRCT scanning with its greater ability to visualize fine detail within the lung, has replaced conventional chest radiography as the preferred imaging method for the ILDs. The characteristic radiographic features of many ILDs (especially for IPF) and correlations of these features with histopathology have been described. HRCT has been found useful in the evaluation of ILDs in the following areas: Identification of the presence of disease (often being abnormal when other studies are normal or only mildly impaired), evaluation of the extent of disease, characterization of the patterns of the disease, narrowing the differential diagnosis, as a guide to the site of biopsy and assessing the clinical course of the disease and response to therapy [5].

The main aim of the study was to assess the role of HRCT in the evaluation of interstitial lung disease with special attention to evaluate interstitial lung disease in symptomatic patients with normal or equivocal chest radiograph findings, to accurately assess the pattern, distribution and severity of the disease process for the purpose of treatment and management, to differentiate on HRCT reversible changes from those of irreversible which would determine the future prognosis in such patients and to assess the role of HRCT in predicting response to treatment.

Prone and supine HRCT imaging is often used for the evaluation of patients with suspected idiopathic interstitial pneumonia or with restrictive patterns on pulmonary function testing. It helps in distinguishing dependent density (Atelectasis) from pulmonary inflammation or fibrosis. Window level ranging from (600 to $-700 \mathrm{H} \mathrm{U})$ and width $(+1000$ to $+1500 \mathrm{H} \mathrm{U})$ are critical for proper evaluation of HRCT [6]. For HRCT both inspiratory and expiratory imaging should be obtained whenever possible. In addition to detecting air trapping that cannot be identified on inspiratory images, expiratory images are frequently important in assessing for the presence of ground glass opacity [7, 8]. HRCT is superior to chest radiography in diagnosis of interstitial lung disease. Although chest radiography will be the initial imaging modality in these patients, CT diagnosis is often more correct. It is highly accurate in diagnosis of sarcoidosis, silicosis and lymphangitis carcinomatosis. HRCT is indicated when clinical, radiological and functional findings do not allow a specific diagnosis and should be done in patients before biopsy [9].

\section{Material and Methods}

Study design and setting: Study was done on 50 patients in the Radiodiagnosis Department of our Institution over a period of one year from November 2015 to October 2016. The research proposal was approved by the Institutional Ethics Committee of our Institution.

\section{Inclusion Criteria}

1. Patients suspected of having interstitial lung diseases suspected on chest radiographs,

2. Patients with clinically suspected ILD with normal or equivocal radiographs

3. Known cases of interstitial lung diseases (for quantification of the extent of ILD for the purpose of evaluating effectiveness of treatment).

\section{Exclusion Criteria}

1. Pregnant patients.

2. Young females

3. Below 15 children.

Study Protocol: HRCT chest was performed using a dual slice CT scanner (Somatom Spirit, Siemens Healthcare, Germany) with imaging parameters chosen so as to maximize spatial resolution. Narrow slice thickness of $1 \mathrm{~mm}$ was taken from lung apices to lung bases with interslice distance of $1 \mathrm{~cm}$ resulting in images representative of the lungs. High spatial resolution image reconstruction algorithm will be used with minimal field of vision to minimize the size of the pixel.

Window level of $(-750 \mathrm{H} \mathrm{U})$ and Window width of $(+1500 \mathrm{H} \mathrm{U})$ was used for proper assessment of the patients. Scans were performed at full inspiration in supine position. Prone positioning helped in distinguishing gravity dependent atelectasis in the lung bases seen on supine images from early changes of 
idiopathic lung fibrosis. In patients with suspected airway disease additional scans were obtained during expiration for detection of air trapping. Serological testing was done for the presence of different serum markers like ESR, RF, ANA and Anticentromere antibody for the possibility of connective tissue disorders.
Source of data, variables and statistical methods: The source of the data was the clinical, radiological and biochemical parameters of the 50 patients included in the study and the statistical methods appropriated to the relevant data were applied as discussed in results. The study was based on the comparison of all the data and no inter-observer bias was observed.

\section{Results}

In the present study, the age of the patients ranged from 22 years to 85 years with mean age of 53.5 years, majority of the patients were in age group 21-40 years (38\%) (Table 2). In our study there were 44\% male patients and 56\% female patients (Table 3). The normal architecture of the lung at HRCT is depicted in Figure 1. The commonest presenting clinical feature was dyspnoea on exertion present in $64 \%$ of patients followed by cough which was present in $60 \%$ of cases. Fever was present in $24 \%$ patients while skin thickening and arthralgia was seen in $8 \%$ of patients each. Weight loss and Raynaud's phenomenon was seen in $6 \%$ of patients each. (Table 1).

Table-1: Prevalence of the various clinical features of the patients with interstitial lung diseases

\begin{tabular}{|c|c|c|c|}
\hline S. No & Clinical features & No. of Patients & Percentage (\%) \\
\hline 1 & Dyspnoea on exertion & 32 & 64 \\
\hline 2 & Cough & 30 & 24 \\
\hline 3 & Fever & 12 & 8 \\
\hline 4 & Arthralgia & 4 & 8 \\
\hline 5 & Skin thickening & 4 & 6 \\
\hline 6 & Raynaud's phenomenon & 3 & 4 \\
\hline 7 & Weight loss & 2 & 8 \\
\hline 8 & Chest pain & 4 & 6 \\
\hline 9 & Haemoptysis & 3 & 6 \\
\hline
\end{tabular}

Table-2: Age distribution of patients included in the study

\begin{tabular}{|c|c|c|}
\hline Age group in years & No. of Patients & Percentage (\%) \\
\hline $0-20$ & 0 & 0 \\
\hline $21-40$ & 19 & 38 \\
\hline $41-60$ & 18 & 24 \\
\hline $61-80$ & 12 & 2 \\
\hline $81-100$ & 1 & 36 \\
\hline
\end{tabular}

Table-3: Distribution of patients gender-wise

\begin{tabular}{|c|c|c|}
\hline Sex & No. of Patients & Percentage (\%) \\
\hline Male & 22 & 44 \\
\hline Female & 28 & 56 \\
\hline Total & 50 & 100 \\
\hline
\end{tabular}

The most common indication for which HRCT chest was performed was Idiopathic pulmonary fibrosis present in $32 \%$ cases, Rheumatoid arthritis was the next common indication seen in $26 \%$ of patients followed by Scleroderma which was an indication in $20 \%$ cases. SLE was an indication in $18 \%$ of patients while occupational ILD and Mycosis Fungoides was an indication in $2 \%$ patients each (Fig. 4). X-ray features were present only in 21 (42\%) patients out of 50 patients. There were 35 (70\%) patients in whom serum markers were present. 
Table 4: HRCT findings in patients of various interstitial lung diseases encountered in the study

\begin{tabular}{|c|c|c|c|}
\hline RA & Scleroderma & IPF & SLE \\
\hline Ground glass haze & Septal lines & Septal lines & Septal lines \\
\hline Bronchiectasis & Parenchymal bands & Honeycombing & Subpleural nodules \\
\hline Reticulations & Subpleural nodules & Bronchiectasis & Bronchiectasis \\
\hline Honeycombing & Bronchiectasis & Ground glass haze & Ground glass haze \\
\hline Pleural Effusion & Ground glass haze & Subpleural cysts & Irregular interface \\
\hline & Honeycombing & & Consolidation \\
\hline & Dilated esophagus & & \\
\hline
\end{tabular}

The most common finding in interstitial lung diseases on HRCT was septal lines seen in 21 (42\%) patients followed by bronchiectasis seen in 20 (40\%) patients, ground glass haze was seen in $16(32 \%)$ patients, honeycombing was present in $10(20 \%)$ cases while subpleural nodules were seen in $9(18 \%)$ patients. Consolidation was present in $2(4 \%)$ patients and parenchymal bands were seen in $6(12 \%)$ patients. Subpleural cyst and dilated esophagus was seen in 2 (4\%) patients each while effusion and scar carcinoma was present in $1(2 \%)$ patient each (Table 4$)$.

Figure 1,2 and 3 show the serological and radiological distribution of findings in patients with interstitial lung diseases.

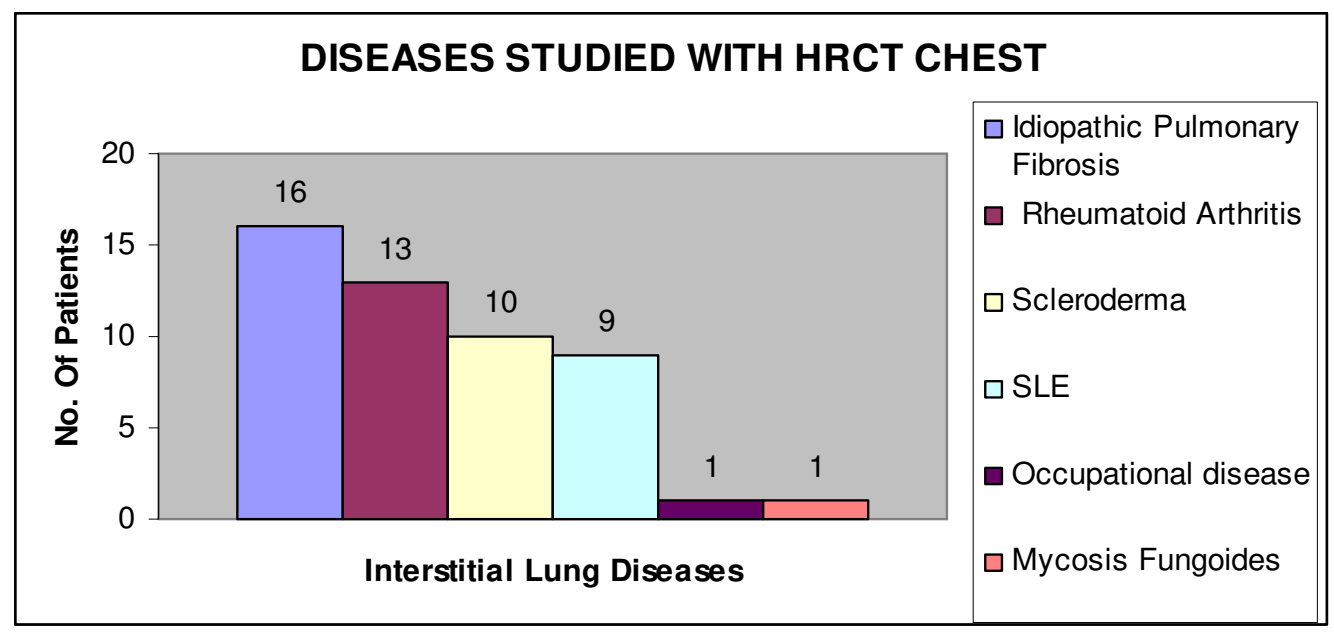

Fig 1: Various diseases encountered during the study

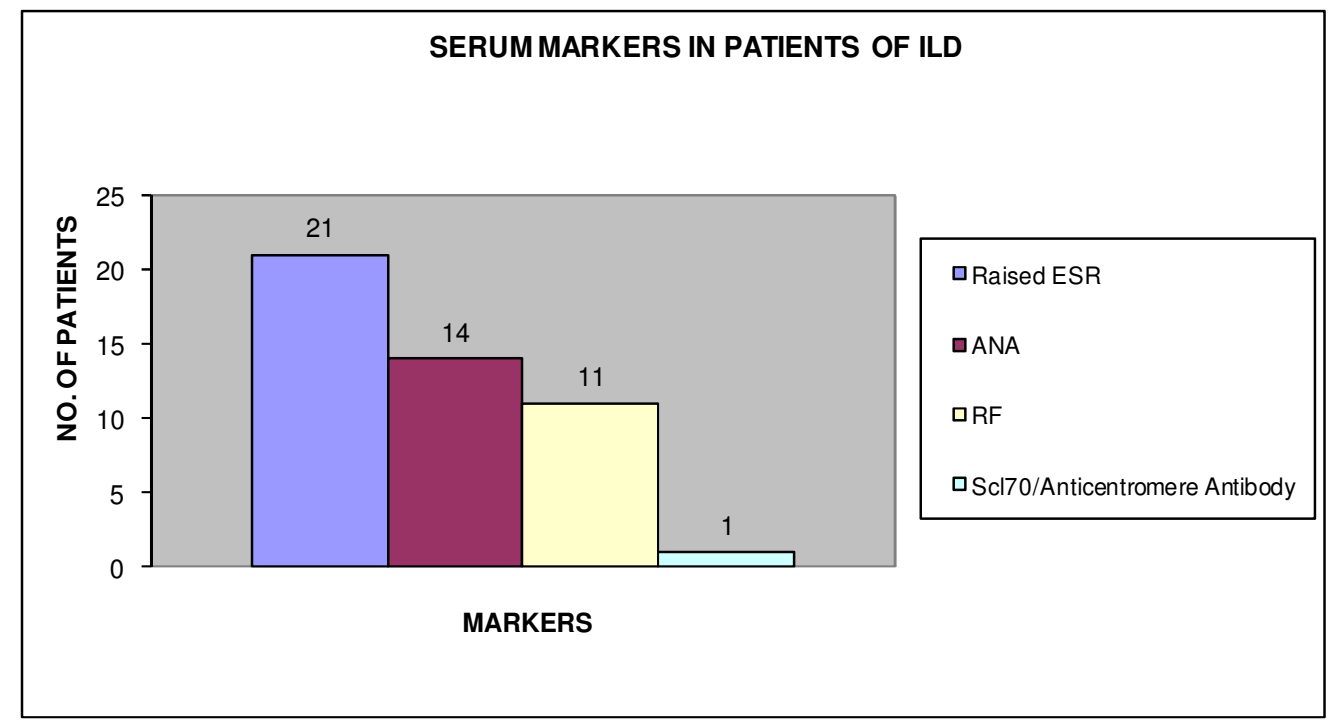

Fig 2: Distribution of serum markers of ILD's in the study 


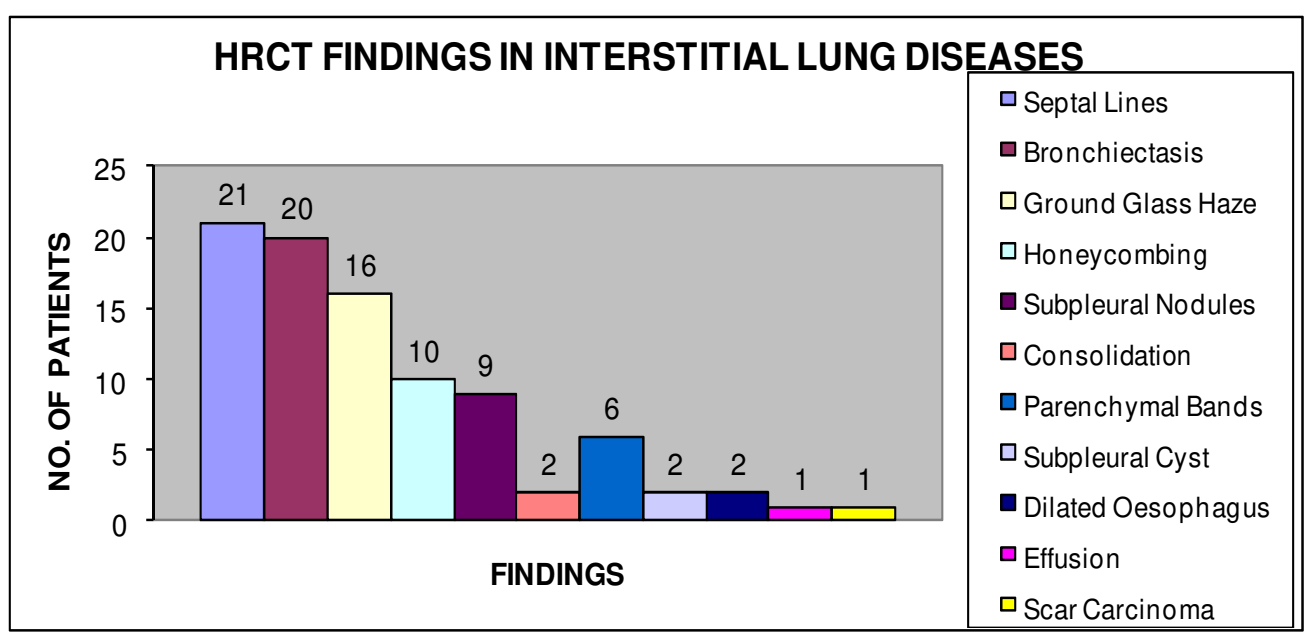

Fig 3: Distribution of HRCT findings in various patients

Rheumatoid Arthritis: The most common HRCT finding in patients of RA was ground glass haze seen in 7 patients followed by bronchiectasis seen in 5 patients, reticulation was present in 3 cases, honey combing was seen in 2 patients and effusion was seen in $1(7.6 \%)$ patient (Fig 3).

Findings of RA were detected in $10(76.9 \%)$ cases on HRCT Chest while only $6(46.2 \%)$ patients showed positive findings on X-Ray Chest. Most common serum marker in RA was raised ESR present in $100 \%$ of patients, RF was present in $10(76.9 \%)$ patients while ANA was present in 1(7.6\%) patient.

Scleroderma: The most common HRCT finding in patients of Scleroderma was septal lines and parenchymal bands present in 5 cases each followed by subpleural nodules and bronchiectasis seen in 4 patients each. Honeycombing, ground glass haze and dilated oesophagus was seen in 2 patients each while scar carcinoma was present in 1 patient (Fig. 1). Findings of Scleroderma were detected in $8(80 \%)$ cases on HRCT Chest while only $5(50 \%)$ patients showed positive findings on X-Ray Chest. Most common serum marker in Scleroderma was ANA present in 6 (60\%) patients while Scl70 was present in $1(10 \%)$ patient.

Systemic Lupus Erythematosis: The most common HRCT finding in patients of SLE was septal lines seen in 4 patients followed by subpleural nodules and bronchiectasis seen in 3 patients each, ground glass haze was seen in 2 patients while irregular interface and consolidation was seen in 1 patient each (Fig. 1). Findings of SLE were detected in 7 (77.8\% patients on HRCT Chest while only $2(22.2 \%)$ patients showed positive findings on X-ray Chest.

Idiopathic Pulmonary Fibrosis: the most common HRCT finding in patients of IPF was septal lines and honeycombing seen in 8 patients each followed by bronchiectasis seen in 7 cases, ground glass haze was seen in 4 patients and subpleural cysts were seen in 2 cases (Fig.3). Findings of IPF were detected in $16(100 \%)$ cases on HRCT Chest while only $7(43.7 \%)$ patients showed positive findings on X-Ray Chest. Only serum marker seen in patients of IPF was raised ESR seen in $8(50 \%)$ of patients. Diagnosis of IPF was made on the basis of clinico-radiological findings and by exclusion of other possibilities. Lung biopsy was advised to the patients for confirmation. Out of 16 patients only $3(19 \%)$ cases underwent biopsy and HRCT findings were confirmed while in $13(81 \%)$ cases who refused for the procedure, follow up was done and clinico- radiological improvement was seen after getting the treatment for IPF.

Mycosis Fungoides: HRCT findings in patient of mycosis fungoides were septal lines, ground glass haze, bronchiectasis and miliary nodules. Findings of mycosis fungoides were present in both X-ray and HRCT Chest. Associated findings in patient of mycosis fungoides were lytic bone lesions and liver cysts (Fig. 3).

Occupational Lung Diseases: The HRCT findings in patient of pneumoconiosis were subpleural consolidation, centrilobular nodules and parenchymal bands. Findings were present in both X-Ray and HRCT chest (Fig.3). 


\section{Discussion}

Interstitial lung diseases also called as diffuse parenchymal lung diseases are a diverse group of pulmonary disorders classified together because of similar clinical, roentgenographic, physiologic or pathologic features. Patients with suspected diffuse interstitial lung disease usually have a chest radiograph as the initial imaging investigation. In majority of patients this is abnormal. In most patients the chest radiographic appearances are not specific while in small proportion of patients with diffuse interstitial lung disease the chest radiograph is normal [5].The limitations of plain chest films in the assessment of lung diseases, especially diffuse interstitial lung diseases and the difficulties of characterizing lung morphology precisely became even more evident when HRCT was introduced as a new tool in radiologic imaging [10]. The components of the HRCT findings that are helpful in the diagnosis of ILD include the pattern of parenchymal abnormality (eg, consolidation, reticular pattern), the anatomic distribution (upper vs lower, central vs peripheral) and associated findings (eg, mediastinal lymphadenopathy) . The most common cause of interstitial lung disease is idiopathic pulmonary fibrosis1. In the present study, the maximum number of cases were seen in association with connective tissue diseases (32 cases) followed by idiopathic pulmonary fibrosis which was present in (16 cases).

Rheumatoid arthritis: Rheumatoid Arthritis (RA) is a connective tissue disease characterized by symmetrical inflammatory arthritis. It is the most common of the connective tissue diseases. The majority of patients have extra articular disorders. RA is associated with a broad spectrum of pleural and pulmonary manifestations. Most, but not all patients with pleuropulmonary disease have other clinical evidence of RA [11]. HRCT is the most sensitive parameter to detect the early interstitial changes in patients of RA. HRCT can show evidence of interstitial lung changes even when clinical and pulmonary function tests are normal. HRCT is superior to plain chest radiograph in the evaluation of early interstitial lung changes associated with RA [12].

HRCT findings of RA associated with interstitial lung diseases observed in current study were, ground glass haze present in 7 cases, bronchiectasis was seen in 5 cases and reticulation was present in 3 cases. Honeycombing was seen in 2 cases and architectural distortion was present in 1 case. In none of the cases nodules were seen but right sided pleural effusion was seen in 1 case. Similar findings were seen in previous studies $[13,14]$.

Scleroderma: Progressive systemic sclerosis (scleroderma) is a connective-tissue disease of unknown pathogenesis that affects 30- to 50-years-old women three times as often as it affects men. This type of sclerosis is characterized by over production of collagen which leads to fibrosis of the lungs, skin, vasculature and visceral organs. Patients present with thickening and tightening of the skin, musculoskeletal manifestations, Raynaud's phenomenon and fibrosis of the lungs, kidneys and gastrointestinal tract [15]. HRCT is much more sensitive than chest radiography when assessing subtle pulmonary involvement in patients of scleroderma. It therefore seems to be the method of choice for evaluation of structural damage to lung parenchyma. 35\% cases of scleroderma had normal Xrays while HRCT detected findings in $91 \%$ cases ${ }^{8}$. Similar observations were made in our study.

HRCT findings in present study revealed predominant lower lobe involvement in 3 cases. Septal lines and parenchymal bands were present in 5 cases each. Ill defined subpleural nodules and bronchiectasis were present in 4 cases each while honeycombing was seen in 2 cases. Ground glass haze and dilated oesophagus was seen in 2 cases each. 1 case had developed carcinoma over fibrotic changes. Findings were similar with various earlier studies $[16,17]$.

Systemic lupus erythematosus: Systemic lupus erythematosus (SLE) is a systemic autoimmune disease. Systemic lupus erythematosus is an autoimmune disease of unknown pathogenesis characterized at histologic examination by deposition of autoantibodies and immune complexes that damage tissues and cells. The presentation is usually systemic and includes fatigue, malaise, anorexia, fever and weight loss. The disease predominantly affects women (F:M, 10:1) aged 20-50 years [18]. In the present study, there were 9 cases of SLE. HRCT findings were interlobular septal thickening present in 4 cases and irregular interfaces in 1 cases. Subpleural nodules and bronchiectasis were seen in 3 cases each, ground glass haze was seen in 2 cases while consolidation was seen in 1 case. No pleural thickening or effusion was seen. In 7 cases where Xrays were normal, HRCT was able to detect the lung 
involvement. HRCT is superior to X-ray in evaluating the involvement of lung in interstitial lung diseases and help detect changes with greater accuracy and confidence than chest radiography. Additionally, thinsection CT is able to define the extent of disease and to identify abnormalities when chest radiographs appeared to have normal finding $[19,20]$.

Idiopathic pulmonary fibrosis: Idiopathic pulmonary fibrosis (IPF) is defined as a specific form of chronic fibrosing interstitial pneumonia of unknown cause, limited to the lungs and associated with a histologic pattern of usual interstitial pneumonia (UIP). It is slightly more common in men and occurs mainly in patients over 50 years old. Clinically, IPF is characterized by the insidious onset of a non productive cough and dyspnoea. The prognosis is poor; the median survival from the time of diagnosis is $2.5-3.5$ years [5].

In the current study, there were 16 cases of IPF. Bilateral parenchymal abnormalities were seen in fifteen cases. Basal and subpleural distribution was seen in thirteen cases. Honeycombing and septal lines were seen in eight cases each. Bronchiectasis was seen in seven cases, ground glass haze was seen in four cases while subpleural cysts were present in two cases. HRCT findings were present in all the sixteen cases while $\mathrm{X}$ Ray findings were present in only seven cases. Similar findings were observed in study conducted by [21]. Out of 16 cases only 3 cases $(18.75 \%)$ underwent lung biopsy and histopathological findings were found to be consistent with those of HRCT findings while 13(81\%) cases showed clinico-radiological improvement after receiving treatment. A clinical diagnosis in conjunction with distinct distribution patterns on HRCT is an effective tool for making the diagnosis of Idiopathic pulmonary fibrosis [22].

Mycosis funoides: Mycosis fungoides, a malignant skin condition with the microscopic appearance of lymphoma, is easily confused with the skin manifestations of leukemia and Hodgkin's disease. It may remain localized to the skin for long periods, but in some cases, it may progress to a systemic stage. The organs most commonly affected are lymph nodes, spleen, liver, lungs, gastrointestinal tract, bones and adrenal glands [23].

In Mycosis Fungoides, HRCT lung reveals areas of ground glass haze with bronchiectasis, septal lines and small nodules [24]. Similar observations were made in our study. Associated systemic findings seen in our study were simple cysts present in liver along with lytic bone lesions. Similar associated findings in mycosis fungoides were reported in some earlier studies [25].

Occupational lung diseases: Numerous occupations expose workers to chemicals, gases, dust and toxins that can damage the lungs. Silicosis, asbestosis, and coalworker's pneumoconiosis all belong to a group called pneumoconiosis. Of these pneumoconioses, silicosis most often occurs in people working in fields involving high exposure to dust. Such people include miners, construction workers, ceramics workers, tunnel drillers, sandblaster and stone carvers. In the present study, HRCT lung revealed multiple confluent areas of subpleural consolidation, centrilobular nodules and interlobular septal thickening as parenchymal bands. Involvement was bilateral and there was upper lobe predominance .This was in accordance with the earlier studies $[26,27]$. In a subset of patients with interstitial lung disease who undergo lung biopsy, accurate diagnosis can be made with HRCT findings alone. Transbronchial and open lung biopsies are commonly avoided because CT helps to form a specific diagnosis with high level of confidence. HRCT made accurate diagnosis in most of the cases [28].

HRCT plays a major role in the assessment of patients who have diffuse lung disease. By eliminating superimposition of structures, CT allows for a better assessment of the type, distribution and severity of parenchymal abnormalities than is possible with chest radiographs. HRCT currently has the best sensitivity and specificity of any imaging method for the assessment of focal and diffuse lung diseases. By demonstrating the pattern and distribution of these abnormalities, HRCT often allows for a confident diagnosis to be made. Thus, HRCT is indicated in patients with suspected diffuse infiltrative lung disease who have normal or questionable radiographic findings [4].

\section{Conclusion}

In conclusion, HRCT is a valuable technique for evaluating extent of lung involvement in various interstitial lung diseases even when chest X-rays are normal. It is capable of imaging the lung with excellent spatial resolution and provides good anatomic detail. Specific diagnosis can be made and is useful in planning patient's management. In conjunction with 
clinical diagnosis; it can obviate the need for lung biopsy.

Conflicts of interest: There are no financial or any other conflicts of interest between the authors.

The manuscript has been read and agreed upon by all the authors for submission to your journal.

\section{Funding: Nil, Conflict of interest: None Permission of IRB: Yes}

\section{References}

1. Ryu JH, Daniels CE, Hartman TE, Yi ES. Diagnosis of interstitial lung diseases. Mayo Clin Proc. 2007 Aug;82(8):976-86.

2. Fulmer JD. The interstitial lung diseases. Chest. 1982 Aug;82(2):172-8.

3. Meziane MA, Hruban RH, Zerhouri EA, Wheeler EA, Khouri NF, Fishman EK et al. High Resolution Computed Tomography of the lung parenchyma with pathological correlation. Radiology 1988; 8(1): 27-54.

4. Müller NL. Computed tomography and magnetic resonance imaging: past, present and future. Eur Respir J Suppl. 2002 Feb;35:3s-12s.

5. King TE Jr. Clinical advances in the diagnosis and therapy of the interstitial lung diseases. Am J Respir Crit Care Med. 2005 Aug 1;172(3):268-79. Epub 2005 May 5.

6. Gotway MB, Reddy GP, Webb WR, Elicker BM, Leung JW. High-resolution CT of the lung: patterns of disease and differential diagnoses. Radiol Clin North Am. 2005 May;43(3):513-42, viii.

7. Brody AS. Imaging considerations: interstitial lung disease in children. Radiol Clin North Am. 2005 Mar;43(2):391-403.

8. Schurawitzki H, Stiglbauer R, Graninger W, Herold C, Polzleitner D, Burghuber OC et al. Interstitial lung disease in progressive Systemic Sclerosis: High resolution CT versus Radiography. Radiology 1990;176(3):755-759.

9. Mathieson JR, Mayo JR, Chatherine AS, Muller NL. Chronic diffuse infiltrative lung disease: Comparison of diagnostic accuracy of CT and chest radiography. Radiology 1989; 171(1):111-116.
10. Müller NL. Clinical value of high-resolution CT in chronic diffuse lung disease. AJR Am J Roentgenol. 1991 Dec;157(6):1163-70.

11. Lamblin C, Bergoin C, Saelens T, Wallaert B. Interstitial lung diseases in collagen vascular diseases. Eur Respir J Suppl. 2001 Sep;32:69s-80s.

12. Raniga S, Sharma P, Kaur G, Arora A, Khalasi Y, Vohra PV. Interstitial lung disease in Rheumatoid Arthritis. Study of Thirty Cases: Ind J radiol Imag 2006;16(4):835-839.

13. Remy-Jardin M, Giraud F, Remy J, Copin MC, Gosselin B, Duhamel A. Importance of ground-glass attenuation in chronic diffuse infiltrative lung disease: pathologic-CT correlation. Radiology. 1993 Dec;189(3):693-8.

14. Tanaka N, Kim JS, Newell JD, Brown KK, Cool CD, Meehan R, Emoto T, Matsumoto T, Lynch DA. Rheumatoid arthritis-related lung diseases: CT findings. Radiology. 2004 Jul;232(1):81-91. Epub 2004 May 27.

15. Mayberry JP, Primack SL, Müller NL. Thoracic manifestations of systemic autoimmune diseases: radiographic and high-resolution CT findings. Radiographics. 2000 Nov-Dec;20(6):1623-35.

16. Goldin JG, Lynch DA, Strollo DC, Suh RD, Schraufnagel DE, Clements PJ, Elashoff RM, Furst DE, Vasunilashorn S, McNitt-Gray MF, Brown MS, Roth MD, Tashkin DP; Scleroderma Lung Study Research Group. High-resolution CT scan findings in patients with symptomatic scleroderma-related interstitial lung disease. Chest. 2008 Aug;134(2):358-67. doi: 10.1378/chest.07-2444. Epub 2008 Jul 18.

17. Strollo D, Goldin J. Imaging lung disease in systemic sclerosis. Curr Rheumatol Rep. 2010 Apr;12(2):156-61. doi: 10.1007/s11926-010-0095-0.

18. Strange C, Highland KB. Interstitial lung disease in the patient who has connective tissue disease. Clin Chest Med. 2004 Sep;25(3):549-59, vii.

19. Fenlon HM, Doran $M$ and Sant SM. HRCT in systemic lupus erythematosus. American Journal of Roentgenology 1996; 166(2):301-307.

20. Kakati S, Doley B, Pal S, Deka UJ. Pulmonary manifestations in systemic lupus erythematosus (SLE) 
with special reference to HR CT. J Assoc Physicians India. 2007 Dec;55:839-41.

21. Müller NL, Staples CA, Miller RR, Vedal S, Thurlbeck WM, Ostrow DN. Disease activity in idiopathic pulmonary fibrosis: CT and pathologic correlation. Radiology. 1987 Dec;165(3):731-4.

22. Souza CA, Müller NL, Flint J, Wright JL, Churg A. Idiopathic pulmonary fibrosis: spectrum of highresolution CT findings. AJR Am J Roentgenol. 2005 Dec;185(6):1531-9.

23. Shapeero LG, Young SW. Mycosis fungoides: manifestations on computed tomography. Radiology. 1983 Jul;148(1):202.

24. Ueda T, Hosoki N, Isobe K, Yamamoto S, Motoori K, Shinkai H. Diffuse pulmonary involvement by Mycosis Fungoides: HRCT and Pathological findings. Journal of Thoracic Imaging 2002;17(2):157-159.
25. Byun JH, Ha HK, Kim AY, Kim TK, Ko EY, Lee JK, Yu ES, Myung SJ, Yang SK, Jung HY, Kim JH. CT findings in peripheral T-cell lymphoma involving the gastrointestinal tract. Radiology. 2003 Apr;227(1):5967. Epub 2003 Feb 11.

26. Kim KI, Kim CW, Lee MK, Lee KS, Park CK, Choi SJ, Kim JG. Imaging of occupational lung disease. Radiographics. 2001 Nov-Dec;21(6):1371-91.

27. Sharifian SA, Mehrparvar AH, Mohammadi S. An unusual form of silicosis. Acta Medica Iranica 2007;45(2): 158-160.

28. Swensen SJ, Aughenbaugh GL, Myers JL. Diffuse lung disease: diagnostic accuracy of CT in patients undergoing surgical biopsy of the lung. Radiology. 1997 Oct;205(1):229-34.

\section{How to cite this article?}

Gupta S, Mohd Ilyas, Gupta V, Dev G. High resolution computed tomography in the evaluation of interstitial lung diseases: Imaging perspective revisited with review of literature. Int J Med Res Rev 2017;5(04):412-420. doi:10.17511/ijmrr. 2017.i04.07. 